\title{
A Relay Feedback Structure for Time-Delay Processes under Disturbances: Stability Analysis using Poincaré map *
}

\author{
Moisés T. da Silva* Péricles R. Barros* \\ * Electrical Engineering Department, Federal University of Campina \\ Grande, Campina Grande PB, 58429-900, Brazil (e-mail: \\ moises.silva@ee.ufcg.edu.br, prbarros@dee.ufcg.edu.br).
}

\begin{abstract}
In this paper, the stability of a relay feedback structure that provides a stable and symmetrical oscillation for process under disturbances is analysed. The relay feedback structure consists of a block which removes static disturbance or drift followed by a relay. The block is composed of a high-pass filter followed by a relay plus an integrator. From an equivalent relay structure, the conditions for the existence and local stability of the limit cycle for the relay feedback structure and a linear time-invariant (LTI) system with time delay are obtained. In addition, the analysis is extended to cases where the time delay is larger than half of the oscillation period. Simulation studies illustrate the results.
\end{abstract}

Keywords: Relay feedback, Poincaré map, disturbance, stability analysis.

\section{INTRODUCTION}

The relay feedback method, proposed by Åström and Hägglund (1984), has been widely used since its introduction in the 1980's. For instance, the relay experiments are applied to multivariate process control (Wang et al., 1997), relay auto-tuning (Atherton, 2006) and system identification (Liu et al., 2013).

For processes under the effect of disturbances, the standard relay feedback method results in errors in the ultimate data estimate. Many relay feedback methods have been proposed in order to overcome these errors (Park et al., 1997; Sung and Lee, 2006; Sung et al., 2006; Friman and Waller, 1997; Lee et al., 2011). As an alternative, a relay feedback structure has been proposed in da Silva and Barros (2017) which guarantees a stable and symmetrical oscillation under static disturbance or drift.

In several cases, limit cycle oscillations (a nontrivial periodic orbit that is isolated) occur in relay feedback systems. The limit cycle is symmetric if the periodic solution has equal time intervals between the positive and negative switching, and unimodal if the relay switches two times per period.

Limit cycle oscillations are an important phenomenon in control design because it usually imposes undesirable effects on the system. In Åström (1995), using a Poincaré map, conditions for limit cycles in linear systems with time delays and relay feedback have been obtained. In Johansson et al. (1999), the characterization of relay feedback systems that have multiple fast switches is investigated. Alternatively, in Varigonda and Georgiou (2001) the conditions for the existence and local stability of the limit cycle

\footnotetext{
‡ This work was supported in part by the CAPES (Coordenação de Aperfeiçoamento de Pessoal de Nível Superior).
}

for a relay with hysteresis are provided. The conditions for global stability in relay feedback systems were presented in Gonçalves et al. (2001) and Varigonda and Georgiou (2000).

In da Silva and Barros (2018), the relay feedback structure for processes under disturbances was analyzed using the Poincaré map and a state-space representation for processes with no time delay. In this paper, the analyses of this relay feedback structure are extended to processes with time delay. In order to simplify the analysis of the relay feedback structure under study, an equivalent relay structure is obtained. For the equivalent structure and LTI system with time delay, the conditions for the existence of the limit cycle are established. Furthermore, using the Jacobian of the corresponding Poincaré map, the local stability of the limit cycle is investigated.

This paper is organized as follows: in Section II, the relay feedback structure for processes under static disturbance or drift is presented; in Section III, an equivalent relay structure is obtained in order to simplify the analysis; in Section IV, the analysis of the existence and local stability of the limit cycle for the equivalent relay feedback structure are presented; in Section V, simulation study are shown; in Section VI conclusions are discussed.

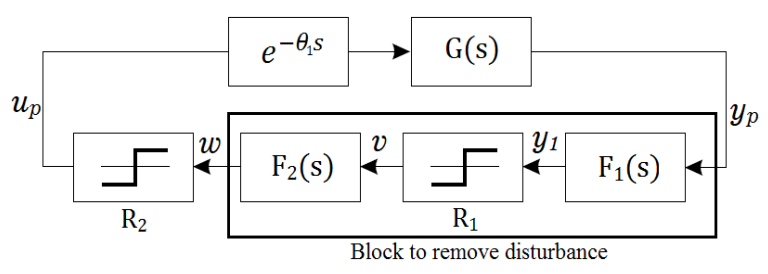

Figure 1. Schematic diagram of the relay feedback structure. 


\section{THE RELAY FEEDBACK STRUCTURE}

Consider a single-input single-output (SISO) LTI system satisfying the following linear dynamic equations

$$
\left\{\begin{array}{l}
\dot{x}_{p}(t)=A x_{p}(t)+B u_{p}\left(t-\theta_{1}\right) \\
y_{p}(t)=C x_{p}(t)
\end{array}\right.
$$

where $x_{p} \in \mathbb{R}^{n}, A \in \mathbb{R}^{n \times n}, B \in \mathbb{R}^{n \times 1}, C \in \mathbb{R}^{1 \times n}$ and $A$ is Hurwitz. The system, without the time delay, can also be described by the transfer function

$$
G(s)=C(s I-A)^{-1} B .
$$

The relay feedback structure of interest is shown in Fig. 1. This structure is composed of two blocks. The first block is used to remove static disturbance or drift and consists of a high-pass filter $F_{1}(s)$, followed by a relay $\left(R_{1}\right)$ and a low-pass filter $F_{2}(s)$. The second block is a standard relay $\left(R_{2}\right)$.

Consider the input and output signals of the relays as $m(t)$ and $z(t)$, respectively. The relays are defined as

$$
z(t) \in\left\{\begin{array}{c}
\{-1\} \text { if } m(t)>\varepsilon, \text { or } m(t)>-\varepsilon \\
\text { and } z\left(t_{-}\right)=-1 \\
\{1\} \text { if } m(t)<-\varepsilon, \text { or } m(t)<\varepsilon \\
\text { and } z\left(t_{-}\right)=1 \\
\{-1,1\} \text { if } m(t)=-\varepsilon \text { and } z\left(t_{-}\right)=-1, \\
\text { or } m(t)=\varepsilon \text { and } z\left(t_{-}\right)=1
\end{array}\right.
$$

where $\varepsilon \geq 0$ is the hysteresis parameter.

The high-pass filter $F_{1}(s)$ is used to remove the static disturbance or drift, and is chosen as

$$
F_{1}(s)=1-e^{-s \tau_{d}}
$$

where $\tau_{d}$ is the filter time constant and should be chosen sufficiently small.

The low-pass filter $F_{2}(s)$ is chosen as an integrator, i.e.

$$
F_{2}(s)=\frac{1}{s}
$$

The standard relay feedback method cannot provide a stable oscillation under large static disturbances or drift. The relay feedback structure under study can be used to overcome this limitation. Thus, a limit cycle insensitive to disturbances is obtained.

Assuming the phase angle of the filter $F_{1}(s)$ is $\theta_{1}$ at the frequency $\omega$. The describing function for the relay structure is given by

$$
N(A, \omega)=\frac{4 h}{\pi A} e^{j\left(\theta_{1}(\omega)-\pi / 2\right)} .
$$

where $h$ is the amplitude of the relay and $A$ is the amplitude of the process output.

The describing function analysis shows that the proposed structure does not change the oscillation characteristics of the relay feedback system. However, only an approximate analysis is provided. A more precise analysis is obtained here using Poincaré map.
More details of the relay feedback structure for processes under static disturbance or drift can be found in da Silva and Barros (2017).

\section{EQUIVALENT RELAY FEEDBACK STRUCTURE}

In order to simplify the analysis of the existence and local stability of the limit cycle for the relay feedback structure shown in Fig. 1, an equivalent structure is obtained as follow.

Lemma 1. Consider the relay feedback structure shown in Fig. 1. Assume the oscillation period for this relay feedback structure equal to $T$ seconds. The transfer function $F_{1}(s)$ is given by (4), and $F_{2}(s)$ is given by (5). Then, except for the initial transient, the relay feedback structure shown in Fig. 3 has the same oscillation period $T$ of the structure presented in Fig. 1, with $\theta_{0}$ equal to $T / 4$.

Proof. The proof of the Lemma is divided into two parts.

Part 1: Start from the relay feedback structure shown in Fig. 1, and assume the oscillation period equal to $T$ seconds. The frequency response for $F_{2}(s)=1 / s$, at the frequency $\omega_{u}$, is given by

$$
F_{2}\left(j \omega_{u}\right)=\frac{1}{\omega_{u}} e^{-j \pi / 2} .
$$

For the input signal $V(s)$, the output of $F_{2}(s)$ is

$$
W\left(j \omega_{u}\right)=\frac{V\left(j \omega_{u}\right)}{\omega_{u}} e^{-j \pi / 2}
$$

Without loss of generality, consider equal amplitudes for both relays. Also consider a square wave $(v(t))$ introduced in the input of the block $F_{2}(s)$. From Eq. (7), there is a $90^{\circ}$ lag between $v(t)$ and $w(t)$ signals. Since the $R_{2}$ relay is a symmetric nonlinearity, without hysteresis, there is no lag between $w(t)$ and $u_{p}(t)$. Thus, there is a $90^{\circ}$ lag between $v(t)$ and $u_{p}(t)$ signals.

Therefore, except for the initial transient, the relay feedback structure shown in Fig. 2, with $\theta_{0}$ equal to $T / 4$, has the same oscillation period $T$ of the structure shown in Fig. 1.

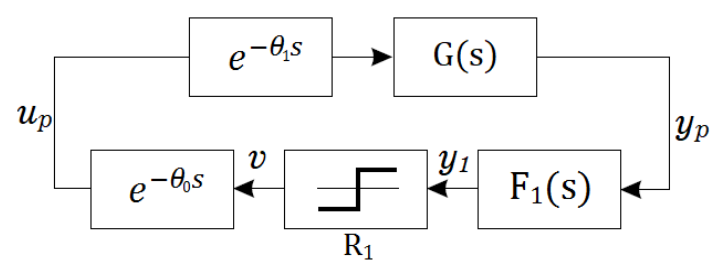

Figure 2. Schematic diagram of the equivalent relay feedback structure.

Part 2: Now from the relay feedback structure in Fig. 2 , by a straightforward application of the superposition principle, the transfer function $F_{1}(s)$ can be displaced to the input of the LTI system, since $F_{1}(s)$ and the system given by (2) are linear.

Thus, the final equivalent relay feedback structure shown in Fig. 3 will oscillate with the same period $T$ of the system shown in Fig. 1. 


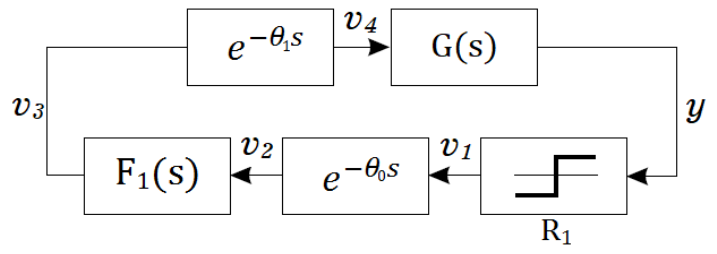

Figure 3. Schematic diagram of the final equivalent relay feedback structure.

Note that the transfer function of linear part of Fig. 3 is given by

with $\theta_{T}=\theta_{0}+\theta_{1}$.

$$
H(s)=G(s) F_{1}(s) e^{-\theta_{T} s},
$$

In order to illustrate the first part of Lemma 1, consider the following process

$$
G(s)=\frac{1}{(s+1)^{2}} e^{-1 s} .
$$

This process is simulated for the relay feedback structure, shown in Fig. 1, the filter time constant is $\tau_{d}=0.01$, magnitude of the relay $M=1$ and hysteresis $\varepsilon=0$. The oscillation period of the process is $T=4.86 \mathrm{~s}$. A periodic solution to this system is shown in Fig. 4. As it can be seen, there exists a $90^{\circ}$ lag between the signals $v(t)$ and $u_{p}(t)$.
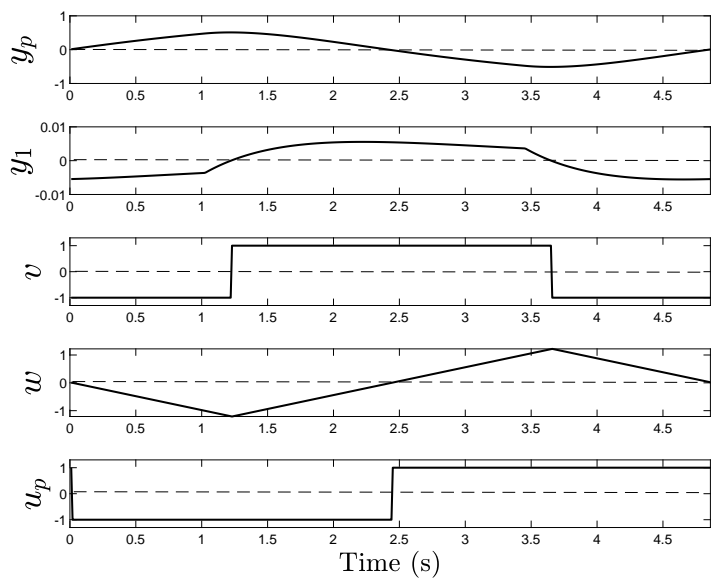

Figure 4. Periodic solution of the relay feedback structure for the system (9).

The second part of Lemma 1 is also illustrated for the process given by (9). A periodic solution to this system is shown in Fig. 5, where $h^{*}=T / 2$ and $\theta_{0}=T / 4=1.215 \mathrm{~s}$.

Note that the signal $v_{2}(t)$ is a delayed version of the relay output signal $v_{1}(t)$. The signal $v_{3}(t)$ is similar a derivative of signal $v_{2}(t)$, and assumes the values of \pm 2 . Whereas, the signal at the input of the linear system $\left(v_{4}(t)\right)$ is a delayed version of the signal $v_{3}(t)$.

The aim of the equivalent structure is to obtain, in steady state, the same periodic solution of the original structure of the relay (Note that the oscillation period of $y_{p}$ in Fig. 4 is the same as the output signal $y$ in Fig. 5). Thus, the difference of the initial transient between the equivalent and original structures does not invalidate the analysis performed in the following sections.

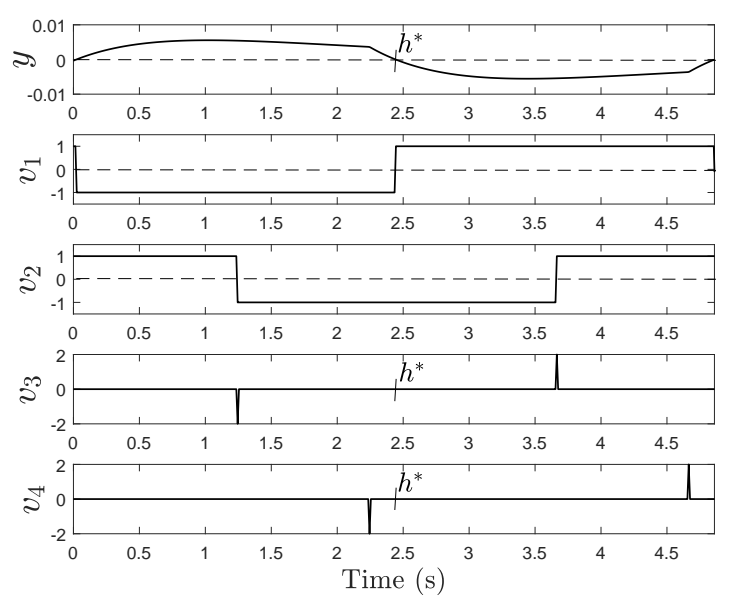

Figure 5. Periodic solution of the final equivalent relay feedback structure for the system (9).

\section{EXISTENCE AND STABILITY ANALYSIS}

In this section, the conditions for the existence of unimodal and symmetrical limit cycles of the equivalent relay feedback structure shown in Fig. 3 are presented. The system under analysis can be represented as

$$
\left\{\begin{array}{l}
\dot{x}(t)=A x(t)+B v_{4}(t) \\
y(t)=C x(t)
\end{array}\right.
$$

in which $A, B$ and $C$ are the same as the original system represented in Eq. (2) and $v_{4}$ is the process input.

The system represented in Eq. (10) can be written in more details as

$$
\left\{\begin{array}{l}
\dot{x}(t)=A x(t)+B\left[v_{1}\left(t-\theta_{T}\right)-v_{1}\left(t-\theta_{T}-\tau_{d}\right)\right] \\
y(t)=C x(t)
\end{array}\right.
$$

For the simplified system, the analysis of the existence of the limit cycle is equivalent to the analysis presented in Varigonda and Georgiou (2001). Thus, the switching surface, composed of a hyperplane of dimension $n-1$, is defined as

$$
S=\left\{x \in \mathbb{R}^{n}: C x=\varepsilon\right\}
$$

The switching surface is a hyperplane that contains the origin and divides the state space into two distinct regions. In one of them $R^{-}=\left\{x \in \mathbb{R}^{n}: C x>\varepsilon\right\}$, in which the system is given by $\dot{x}=A x-2 B$. In the other region $R^{+}=\left\{x \in \mathbb{R}^{n}\right.$ : $C x<\varepsilon\}$, where the system is given by $\dot{x}=A x+2 B$.

Note that for the LTI system under analysis it is correct to consider only the cases $h^{*}>\theta_{0}$, because the $\theta_{0}$ value limits the period of oscillation of the system (the oscillation period is never smaller than the time delay $\theta_{0}$ ). Thus, the periodic solution obtained by the equivalent structure is the same as the original structure.

For the case $h^{*}>\theta_{T}>\theta_{0}$, illustrated in Fig. 5, note that the switching occurs at $t=\theta_{T}+\tau_{d}$, and according to Eq. (11), $v_{4}(t)$ assumes the values of \pm 2 .

When the time delay is larger than half of the oscillation period, $h^{*}$, one or more switches occur between $-\theta_{T} \leq t<$ 0 . This case is illustrated in Fig. 6 for the process: 


$$
G(s)=\frac{1}{s} e^{-1.25 s} .
$$

This process is simulated for the relay feedback structure, shown in Fig. 1, the filter time constant is $\tau_{d}=0.01$, magnitude of the relay $M=1$ and hysteresis $\varepsilon=0$. There is a stable limit cycle with period $T=5.04 \mathrm{~s}$. Thus, $\theta_{0}=$ $T / 4=1.26 \mathrm{~s}$ and $\theta_{T}=2.51 \mathrm{~s}$. As pointed by Åström (1995), also there is an unstable limit cycle with period $T=1 \mathrm{~s}$.

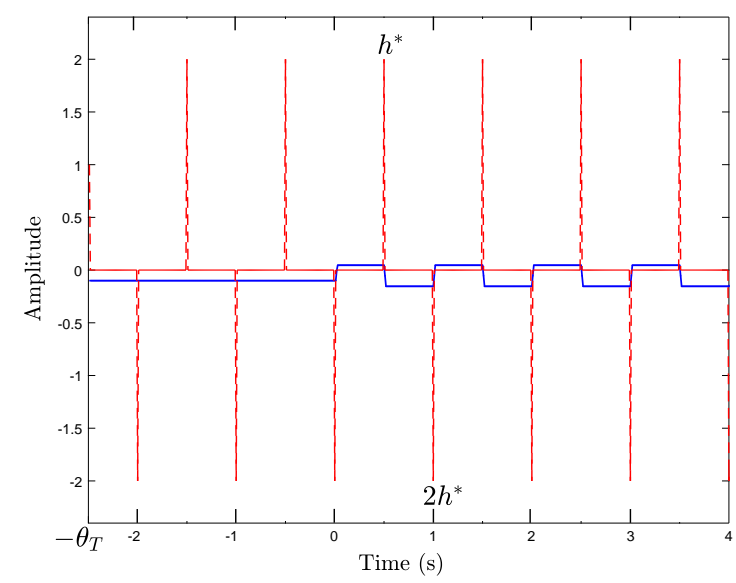

Figure 6. Process input $\left(v_{4}(t)\right.$ - Dashed line) and output $(y(t)$ - Solid line) of the final equivalent relay feedback structure for the case $h^{*}<\theta_{T}$.

For the unstable limit cycle, $h^{*}=0.5 \mathrm{~s}$. Note that $h^{*}<\theta_{T}$, and the linear system input switching occurs at $\Delta \theta=$ $\theta_{T}-n h^{*}=0.51 \mathrm{~s}$, where $n=4$ is the number of switches occurring between $-\theta_{T} \leq t<0$. This process will be discussed in detail in Section 5.

Note also that for $-\theta_{T}<t<0$, the solution can not be obtained by specifying only the initial condition of the linear system, it is necessary also specify an initial solution, $x(\tau)$.

The following theorem is used to identify limit cycles of the relay feedback structure under study, even in cases where the total system delay is larger than half of the oscillation period.

Theorem 1. Consider the linear system given by (10) connected in feedback with the relay given by $(3)$. There is a symmetrical and unimodal limit cycle with period $T=2 h^{*}$ if and only if the following conditions are satisfied:

$$
\begin{aligned}
& \text { (i) } g_{\theta}\left(h^{*}\right) \triangleq(-1)^{n+1} 2 C\left(e^{A h^{*}}+I\right)^{-1}\left(\int_{\Delta \theta}^{\Delta \theta+\tau_{d}} e^{A\left(h^{*}-\tau\right)} B d \tau\right)=\varepsilon, \\
& \text { (ii) } y(t)=C x(t)>\varepsilon, \forall t \in\left(0, h^{*}\right),
\end{aligned}
$$

where

$$
x^{*}=(-1)^{n+1} 2\left(e^{A h^{*}}+I\right)^{-1}\left(\int_{\Delta \theta}^{\Delta \theta+\tau_{d}} e^{A\left(h^{*}-\tau\right)} B d \tau\right),
$$

is the point where the trajectory intercepts the switching surface $S$, and $\Delta \theta=\theta_{T}-n h^{*}$, with the largest integer such that $n h^{*}<\theta_{T}$ ( $n$ is the number of switches occurring in the signal $v_{4}(t)$ between $\left.-\theta_{T} \leq t<0\right)$. For the periodic solution, it is necessary an initial solution, $x(\tau),-\theta_{T} \leq \tau<0$ with $x(0)=x^{*}$.

Proof. The proof is divided into two parts.
Necessity: Assume that there exists a symmetrical limit cycle in the system of Fig. 3.

Case 1: Consider the case $h^{*}>\theta_{T}>\theta_{0}$, where $\theta_{T}=\theta_{0}+\theta_{1}$. For the process represented in Eq. (9) and based on Fig. 5 , the solution of the LTI system, Eq. (10), to $x \in R^{-}$is given by

$$
\begin{aligned}
x\left(h^{*}\right) & =e^{A h^{*}} x(0)+\int_{0}^{h^{*}} e^{A\left(h^{*}-\tau\right)} B v_{3}\left(\tau-\theta_{1}\right) d \tau \\
& =e^{A h^{*}} x(0)+\int_{\theta_{T}}^{\theta_{T}+\tau_{d}} e^{A\left(h^{*}-\tau\right)} B v_{4}(\tau) d \tau .
\end{aligned}
$$

Thus, the proof follows as in Åström (1995).

Case 2: Consider now the case $h^{*}<\theta_{T}$, where $\theta_{T}=\theta_{0}+$ $\theta_{1}$. In this case, the signal at the input of the linear system depends on the number of previous switches, $n$, with $\Delta \theta=\theta_{T}-n h^{*}$ (see Fig. 6). For even $n$ :

$$
\begin{aligned}
x\left(h^{*}\right) & =e^{A h^{*}} x(0)+\int_{0}^{h^{*}} e^{A\left(h^{*}-\tau\right)} B v_{4}(\tau) d \tau \\
& =e^{A h^{*}} x(0)+2 \int_{\Delta \theta}^{\Delta \theta+\tau_{d}} e^{A\left(h^{*}-\tau\right)} B d \tau .
\end{aligned}
$$

For odd $n$, then

$$
\begin{aligned}
x\left(h^{*}\right) & =e^{A h^{*}} x(0)+\int_{0}^{h^{*}} e^{A\left(h^{*}-\tau\right)} B v_{4}(\tau) d \tau \\
& =e^{A h^{*}} x(0)-2 \int_{\Delta \theta}^{\Delta \theta+\tau_{d}} e^{A\left(h^{*}-\tau\right)} B d \tau .
\end{aligned}
$$

Again, the rest of the proof follows as in Åström (1995).

Sufficiency: Assume that $g\left(h^{*}\right)=\varepsilon$, thus $x^{*} \in S$. Also assume $y(t)>\varepsilon$ for $t \in\left(0 ; h^{*}\right)$, thus $v_{4}(t)=-2, t \in\left(0 ; h^{*}\right)$. Therefore, the trajectory from $x^{*}$ will not reach $S$ again before $h^{*}$. It is possible to show that $x(t)$ reaches $S$ after $h^{*}$ seconds on $-x^{*}$, which causes $v_{4}(t)$ to switch to 2 . With a similar argument, it is shown that $x(t)$ returns to $S$ after $h^{*}$ in $x^{*}$, so that there exists a periodic orbit through $x^{*}$.

Note that the expression of the Poincaré map and LTI systems with no time delay is obtained when $\theta_{1}=0$.

In order to analyze using the equivalent structure, it is necessary to know in advance the oscillation period of the original relay structure shown in Fig. 1. In this paper, the oscillation period is assumed to be known. How to obtain this period directly from the original relay structure is under study.

The local stability of the limit cycle is obtained by computing the eigenvalues of the Jacobian of the corresponding Poincaré map (Khalil, 1996).

For systems with time delays, the analysis of the stability of the limit cycles is complicated, since the state of the system is infinite dimensional Aström (1995). The problem is simplified for limit cycles with $h^{*}>\theta_{T}$. This result is presented in the following theorem.

Theorem 2. Consider the linear system given by (10) connected in feedback with the relay given by (3). Assume that there is a symmetric periodic solution with $h^{*}>\Delta \theta>\theta_{0}$, 
with $\Delta \theta=\theta_{T}-n h^{*}$ and $\theta_{T}=\theta_{0}+\theta_{1}$. Considering only the case with $n=0$, i.e. $\Delta \theta=\theta_{T}$, the Jacobian of the Poincaré map is given by

$$
W_{\theta}=\left(I-\frac{\omega_{\theta} C}{C \omega_{\theta}}\right) e^{A h^{*}}
$$

where

$$
\omega_{\theta}=e^{A h^{*}} A x^{*}-2\left[e^{A\left(h^{*}-\theta_{T}-\tau_{d}\right)}+e^{A\left(h^{*}-\theta\right)}\right] B .
$$

The limit cycle is locally stable if and only if $W_{\theta}$ has all its eigenvalues inside the unit disk. It will be unstable if $W_{\theta}$ has at least one eigenvalue outside the unit disk.

Proof. The analysis is performed considering a new origin in $h^{*}+\delta h$, with $x(0)=x^{*}+\delta x$. With the new time base, it follows that

$$
\begin{aligned}
x\left(h^{*}+\delta h\right)=e^{A\left(h^{*}+\delta h\right)} x(0) & +\int_{0}^{h^{*}+\delta h} e^{A\left(h^{*}+\delta h-\tau\right)} B v_{4}(\tau) d \tau \\
=e^{A\left(h^{*}+\delta h\right)}\left(x^{*}+\delta x\right) & \\
& \quad+\int_{\theta_{T}}^{\theta_{T}+\tau_{d}} e^{A\left(h^{*}+\delta h-\tau\right)} B(+2) d \tau
\end{aligned}
$$

Thus, the proof follows as in Åström (1995).

\section{EXAMPLES}

In this Section, using simulated examples, the existence and local stability of the limit cycle is evaluated for the relay feedback structure for processes under disturbance. In all cases, the amplitude of the relay is $M=1$ and the filter time constant is $\tau_{d}=0.01$. The simulations are performed with a sampling time of $T_{s}=0.01$.

Example 1: Consider again the second-order plus time delay process given by Eq. (9). With hysteresis $\varepsilon=0$, the oscillation period obtained from the relay feedback simulation of Fig. 1 is $T=4.86 \mathrm{~s}$. Thus, $\theta_{0}=T / 4=1.215 \mathrm{~s}$. Numerical calculations using Theorem 1 gives one zero for positive $h$, i.e. $h^{*}=2.4178$. Thus, $\hat{T}=2 h^{*}=4.84 \mathrm{~s}$.

The initial condition to obtain a periodic solution is

$$
x(0)=\left(\begin{array}{c}
-0.0151 \\
0
\end{array}\right) .
$$

The Jacobian of the Poincaré map can be computed from Theorem 2 as

$$
W_{\theta}=\left(\begin{array}{ll}
0.3046 & 0.3937 \\
0 & 0
\end{array}\right)
$$

The eigenvalues of $W_{\theta}$ are 0 and 0.3046 . It can be concluded that the limit cycle is locally stable according to Theorem 2.

Example 2: Consider again the process given by Eq. (12), where $A=0, B=1$ and $C=1$. From simulation of the relay feedback structure shown in Fig. 1, a stable limit cycle with period $T=5.04 \mathrm{~s}$ is obtained. Thus, $\theta_{0}=T / 4=1.26 \mathrm{~s}$ and $\theta_{T}=2.51 \mathrm{~s}$. Also there is an unstable limit cycle with period $T=1 \mathrm{~s}$.

As mentioned above, for the unstable limit cycle, the number of switches occurring between $-\theta_{T} \leq t<0$ is $n=4$ and $\Delta \theta=0.51$. From numerical calculations of Theorem 1 , Eq. (13):

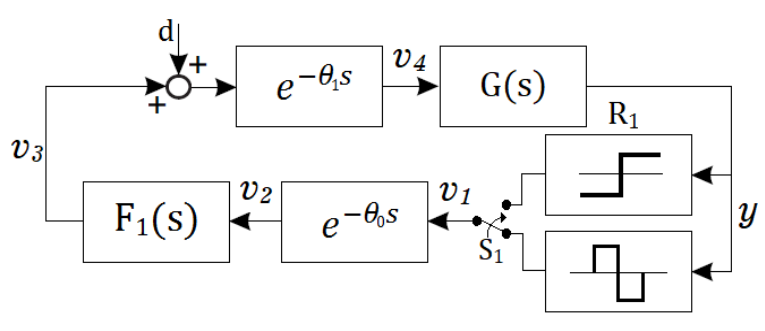

Figure 7. Schematic diagram used for simulation of the equivalent relay feedback structure for process with $h^{*}<\theta_{T}$.

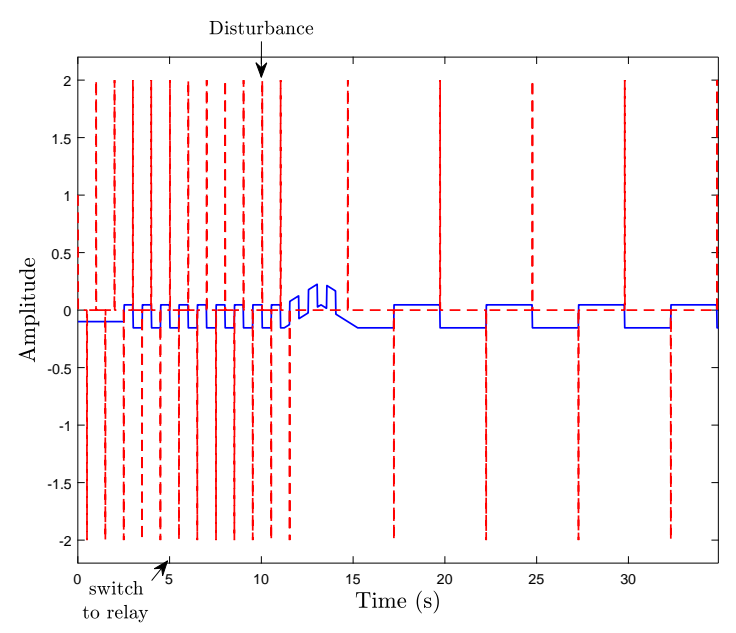

Figure 8. Process input $\left(v_{4}(t)\right.$ - Dashed line) and output $(y(t)$ - Solid line) of the final equivalent relay feedback structure.

$$
\begin{aligned}
g_{\theta}\left(h^{*}\right) & =(-1)^{5} 2 C\left(e^{A h^{*}}+I\right)^{-1}\left(\int_{\Delta \theta}^{\Delta \theta+\tau_{d}} e^{A\left(h^{*}-\tau\right)} B d \tau\right) \\
& =-\tau_{d}=-0.01
\end{aligned}
$$

Thus, the condition $(i)$ is satisfied for a relay with hysteresis $|\varepsilon|=0.01$. In addition, to satisfy the condition (ii) the system should be initialized with $x(\tau)=-0.01$. Thus, as shown in Fig. 6, a limit cycle is obtained with period $T=1 \mathrm{~s}$, which is shorter than the time delay process $\left(\theta_{1}=1.25\right)$.

Using a similar procedure presented in Åström (1995), to obtain the unstable limit cycle $(T=1 \mathrm{~s})$ it is necessary to use the structure shown in Fig. 7. Initially, a double rectangular pulse is applied to the past values of the control signal have been chosen to correspond to the limit cycle. Then, the switch $S_{1}$ is activated and relay $R_{1}$ is applied to input $v_{1}$. The switch to relay feedback structure occurs at time $t=5 \mathrm{~s}$.

The unstable limit cycle is illustrated in Fig. 8, where the simulation in Fig. 6 has been continued for a longer time and at the time $t=10 \mathrm{~s}$ a double rectangular pulse with amplitude $d= \pm 0.01$, period of 4 s and duty cycle of $50 \%$ is introduced into the system. In this case, the solution converges to a stable limit cycle with period $T=5.04 \mathrm{~s}$.

Example 3: Consider the following process

$$
G(s)=\frac{0.2 s^{2}+0.54 s+0.426}{s^{3}+0.25 s^{2}+1.02 s+0.0505} e^{-0.8 s}
$$


With hysteresis $\varepsilon=0.01$, the oscillation period obtained from the relay feedback simulation of Fig. 1 is $T=5.92 \mathrm{~s}$. Thus, $\theta_{0}=T / 4=1.48 \mathrm{~s}$.

In Fig. 9, the solution of $g_{\theta}\left(h^{*}\right)$ is showed. From Theorem 1 , numerical calculations gives two zero for positive $h$ and $|\varepsilon|=0.01$, i.e. $h_{1}^{*}=2.3974$ and $h_{2}^{*}=2.8169$. For $h_{1}^{*}=2.3974$,

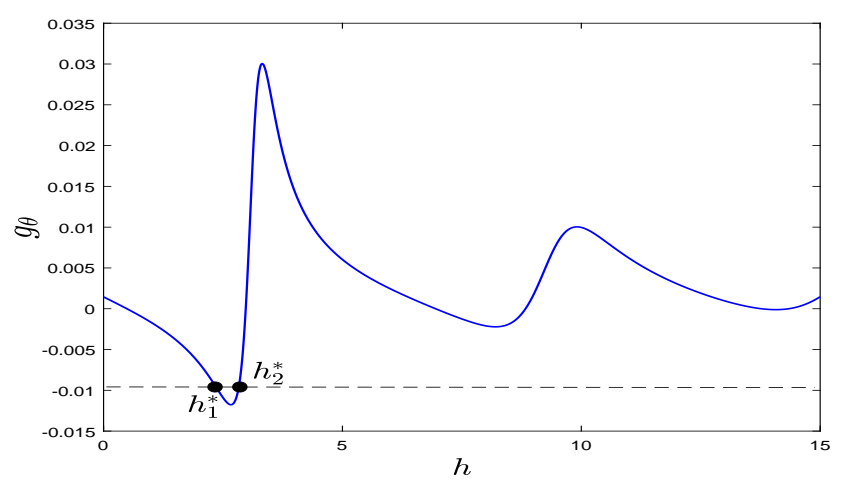

Figure 9. Solution of $g_{\theta}\left(h^{*}\right)$ for Example 3 .

the initial condition to obtain a periodic solution is

$$
x(0)=\left(\begin{array}{c}
-0.0236 \\
0.0202 \\
0.0089
\end{array}\right) .
$$

From Theorem 2, the Jacobian of the Poincaré map can be computed as

$$
W_{\theta}=\left(\begin{array}{lll}
-2.2046 & -0.4352 & -0.7666 \\
-1.9440 & -0.3726 & -1.2264 \\
3.4993 & 0.6766 & 1.9145
\end{array}\right)
$$

The eigenvalues of $W_{\theta}$ are $-1.5788,0.9160$ and 0 . Thus, the limit cycle is locally unstable according to Theorem 2 .

For $h_{2}^{*}=2.8169$, the initial condition to obtain a periodic solution is

$$
x(0)=\left(\begin{array}{c}
-0.0513 \\
0.0066 \\
0.0392
\end{array}\right) .
$$

The Jacobian of the Poincaré map can be computed as

$$
W_{\theta}=\left(\begin{array}{lll}
-0.6442 & -0.2778 & 0.0489 \\
-1.1298 & -0.2921 & -0.7950 \\
1.7345 & 0.5007 & 0.9848
\end{array}\right)
$$

The eigenvalues of $W_{\theta}$ are $-08331,0.8816$ and 0 . According to Theorem 2, it can be concluded that the limit cycle is locally stable.

\section{CONCLUSION}

For a LTI system with time delay, the analysis of symmetric and unimodal limit cycle for a relay feedback structure for processes under disturbances was performed. In order to simplify the analysis, an equivalent structure is obtained. The conditions for the existence of limit cycles are provided using the Poincaré map. The results presented indicate the existence of limit cycles with oscillation period smaller than the system time delay, which implies that such oscillations can only exist if the system has started from this condition by means of an initial solution. The conditions for the local stability of limit cycles was obtained using the Jacobian of Poincaré map. Examples were used to illustrate the analysis.

\section{REFERENCES}

Åström, K.J. (1995). Oscillations in systems with relay feedback. In Adaptive Control, Filtering, and Signal Processing, 1-25. Springer.

Åström, K.J. and Hägglund, T. (1984). Automatic tuning of simple regulators with specifications on phase and amplitude margins. Automatica, 20(5), 645-651.

Atherton, D.P. (2006). Relay autotuning: an overview and alternative approach. Industrial \& engineering chemistry research, 45(12), 4075-4080.

da Silva, M.T. and Barros, P.R. (2017). A relay feedback structure for processes under static disturbances or drift. In Control Technology and Applications (CCTA), 2017 IEEE Conference on, 1171-1176. IEEE.

da Silva, M.T. and Barros, P.R. (2018). Stability analysis of a relay feedback structure for processes under disturbances using poincaré map. In Congresso Brasileiro de Automática (CBA), 2018. SBA.

Friman, M. and Waller, K.V. (1997). A two-channel relay for autotuning. Industrial \& engineering chemistry research, 36(7), 2662-2671.

Gonçalves, J.M., Megretski, A., and Dahleh, M.A. (2001). Global stability of relay feedback systems. IEEE Transactions on Automatic Control, 46(4), 550-562.

Johansson, K.H., Rantzer, A., and Åström, K.J. (1999). Fast switches in relay feedback systems. Automatica, 35(4), 539-552.

Khalil, H.K. (1996). Noninear Systems. Prentice-Hall, New Jersey.

Lee, J., Kim, J.S., Byeon, J., Sung, S.W., and Edgar, T.F. (2011). Relay feedback identification for processes under drift and noisy environments. AIChE Journal, 57(7), 1809-1816.

Liu, T., Wang, Q.G., and Huang, H.P. (2013). A tutorial review on process identification from step or relay feedback test. Journal of Process control, 23(10), 1597-1623.

Park, J.H., Sung, S.W., and Lee, I.B. (1997). Improved relay auto-tuning with static load disturbance. Automatica, 33(4), 711-715.

Sung, S.W. and Lee, J. (2006). Relay feedback method under large static disturbances. Automatica, 42(2), 353356.

Sung, S.W., Lee, J., Lee, D.H., Han, J.H., and Park, Y.S. (2006). Two-channel relay feedback method under static disturbances. Industrial $\mathscr{6}$ engineering chemistry research, 45(12), 4071-4074.

Varigonda, S. and Georgiou, T.T. (2001). Dynamics of relay relaxation oscillators. IEEE Transactions on automatic control, 46(1), 65-77.

Varigonda, S. and Georgiou, T. (2000). Global stability of periodic orbits in relay feedback systems. In Decision and Control, 2000. Proceedings of the 39th IEEE Conference on, volume 4, 3843-3847. IEEE.

Wang, Q.G., Zou, B., Lee, T.H., and Bi, Q. (1997). Autotuning of multivariable pid controllers from decentralized relay feedback. Automatica, 33(3), 319-330. 